NOTA

\title{
OCURRENCIA DE FOCAS (MAMMALIA, CARNIVORA, PHOCIDAE) EN URUGUAY
}

\author{
Eduardo Juri \\ Laboratorio de Mamíferos Marinos, Museo Nacional de Historia Natural, 25 de mayo 582 CP.11.000 \\ Montevideo, Uruguay. Dirección de Coordinación Científica, Instituto Antártico Uruguayo, 8 de Octubre 2958 \\ CP. 11.600 Montevideo, Uruguay. \\ edujuri@gmail.com
}

\section{RESUMEN}

Se presenta una revisión actualizada sobre la ocurrencia de fócidos para Uruguay (1921-2016). Se obtuvieron 64 registros que ocurrieron en la costa Atlántica $(n=45)$, Río de la Plata $(n=13)$ y en otros ríos y arroyos $(n=6)$. Se reclasificaron especímenes erróneamente identificados, además se encontraron especímenes perdidos y se presentan 21 registros de fócidos no publicados.

Palabas clave: Focas, Phocidae, Registros, Uruguay

\section{ABSTRACT}

Occurrence of seals (Mammalia, Carnivora, Phocidae) in Uruguay. An updated review on the occurrence of seals for Uruguay (1921-2016) is presented. A total of 64 records occurred on the Atlantic coast $(n=45)$, Rio de la Plata $(n=13)$ and other rivers and streams $(n=6)$. Erroneously identified specimens were reclassified, missing specimens were found and 21 unpublished seals records are presented.

Key words: Seals, Phocidae, Records, Uruguay

Los pinnípedos (Superfamilia Pinnipedia) se dividen en tres familias dentro del orden Carnivora: Odobenidae (morsas), Otariidae (leones y lobos marinos) y Phocidae (focas). La familia Phocidae la comprenden 19 especies (Jefferson et al., 2015) que tradicionalmente se dividen en cuatro tribus, Monachini (focas monje), Lobodontini (focas antárticas), Cystophorini (focas capuchina y elefantes marinos) y Phocini (el resto de las focas del hemisferio norte; Berta et al., 2006).

En el Océano Atlántico sudoccidental la presencia de focas es ocasional (Silva, 2004), registrándose en las costas de Brasil individuos vagantes de foca leopardo (Hydrurga leptonyx) en los estados de Rio de Janeiro, Santa Catarina y Rio Grande do Sul (Pinedo, 1990; Rosas et al., 1992; Ferreira et al., 1995; Silva, 2004; Moura et al., 2011). La foca cangrejera (Lobodon carcinophaga) se registró en los estados de Rio de Janeiro, Saõ Paulo, Paraná, Santa
Catarina y Rio Grande do Sul (Vaz Ferreira, 1965; Siciliano \& Lodi, 1989; Pinedo, 1990; Ferreira et al, 1995; Simões-Lopes et al., 1995; Silva, 2004; Lodi et al., 2005; Oliveira et al., 2006; Moura et al., 2011). Individuos de elefante marino del Sur (Mirounga leonina) se han registrado tan al norte como en Ilha Fernando de Noronha y en los estados de Sergipe, Bahía, Espírito Santo, Rio de Janeiro, São Paulo, Paraná, siendo más frecuente su presencia en los estados de Santa Catarina y Rio Grande do Sul (Vaz Ferreira, 1965; Carvalho, 1975; Castello, 1984; Lodi \& Siciliano, 1989; Pinedo, 1990; Simões-Lopes et al., 1995; Drehmer et al., 1998; Magalhães et al, 2003; Cherem et al., 2004; Silva 2004; Bastos et al., 2006; Moura et al, 2010; Oliveira et al., 2011).

Para Argentina existen registros de la foca leopardo (H. leptonyx) en las provincias de Buenos Aires, Chubut y Río Negro (Castello \& Rumboll, 1978; Rodríguez et al., 2003; Daneri et al., 2011). La foca de Weddell (Leptonychotes weddellii) se ha reportado en las provincias de Santa Cruz y Tierra del Fuego (Stirling, 1971; Rice, 1998). La foca cangrejera (L. carcinophaga) en las provincias de Buenos Aires, Chubut, Santa Cruz y Tierra del Fuego (Berg, 1898; Scolaro, 1976; Bastida \& Rodríguez, 2006). En Península Valdés, provincia de Chubut ( $\left.42^{\circ} \mathrm{S}\right)$, Argentina se encuentra la única colonia reproductiva continental de elefante marino del Sur ( $M$. leonina) estimada en 42.000 individuos. Ésta colonia muestra un aumento poblacional sostenido (Lewis et al., 1998; McMahon et al., 2005) y en virtud de ese aumento resulta frecuente la presencia de esta especie a lo largo de toda la costa argentina (Bastida et al., 2007).

En Uruguay también ocurren arribos esporádicos de focas antárticas y subantárticas (Ponce de León, 2000), siendo las visitas de M. leonina las más comunes. Las cuatro especies de focas registradas son: foca leopardo $(H$. leptonyx), foca de Weddell $(L$. weddellii), foca cangrejera (L. carcinophaga) y elefante marino del Sur (M. leonina; Achaval et al., 2008; González \& Martínez, 2010). Sin embargo, algunos registros han sido ignorados en las listas mastozoológicas de Uruguay debido a que estos hallazgos no siempre se comunican o son reportados en comunicaciones de difícil acceso. En éste contexto, el objetivo de este trabajo es presentar una revisión 


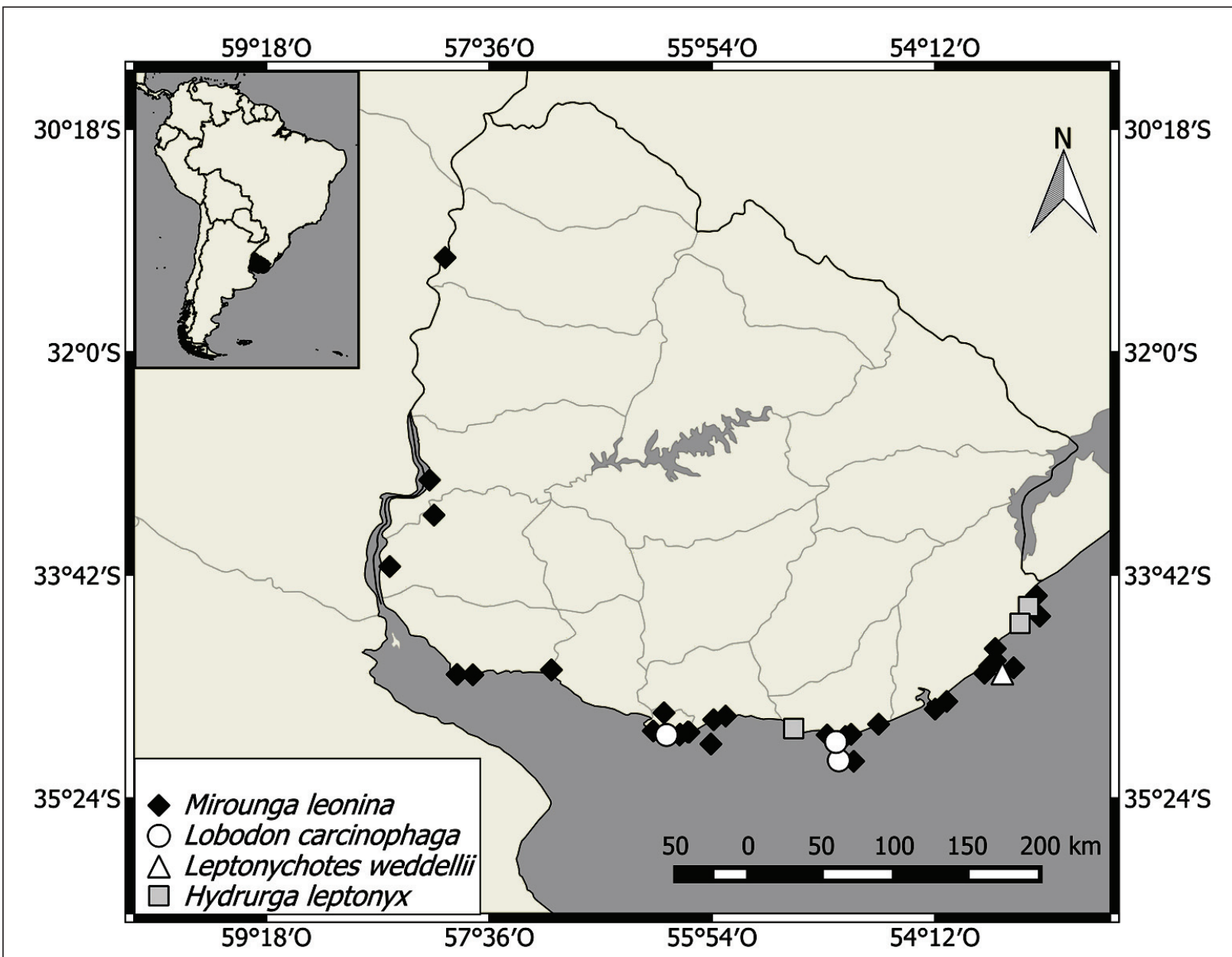

Fig. 1. Localidades de ocurrencia de fócidos registrados en Uruguay.

actualizada sobre la ocurrencia de focas para la costa uruguaya.

Los registros fueron obtenidos de publicaciones científicas, resúmenes presentados en congresos científicos, comunicaciones en la prensa, registros fotográficos, observaciones personales y colecciones que contienen ejemplares procedentes de Uruguay (Zoología de Vertebrados de la Facultad de Ciencias, Museo del Mar de Punta del Este, Museo Nacional de Historia Natural y National Museum of Natural History de Washington D.C.). Se relevaron también las colecciones del Museo Zoológico Dámaso Antonio Larrañaga, Museo de Historia Natural Dr. Carlos Torres de la Llosa y Museo del Colegio Pío, donde no se encontró material de fócidos procedentes de Uruguay. La clasificación del material óseo depositado en colecciones (únicamente cráneos) se realizó siguiendo las guías de González \& Martínez (2010) y Jefferson et al. (2015).

Desde 1921 a 2016 se obtuvieron 64 registros de focas para la costa uruguaya (Tabla1). La mayoría de los registros ocurrieron en la costa Atlántica $(n=45)$ y en menor proporción en el Río de la Plata $(n=13)$, con algunos registros de ingresos a ríos y arroyos $(n=6)$ (Fig. 1).

En este trabajo se reclasificaron algunos especímenes que fueron erróneamente identificados, se encontraron especímenes de colección considerados perdidos hasta el momento, y se presentan 20 registros no publicados de $M$. leonina y uno de $L$. carcinophaga.

La foca leopardo presentó tres registros documentados, en Isla Verde (Rocha) en enero de 1970 (Ximénez \& Langguth, 2008), en Punta Palmar (Rocha) en agosto de 1981 (Vaz Ferreira, 1984) y en Piriápolis (Maldonado) el 18 de setiembre de 2000 (Naya \& Achaval, 2006); de estos individuos no se conserva material en ninguna colección. A excepción de un registro en enero (Ximénez \& Langguth, 2008), los otros dos registros ocurrieron en agosto y setiembre, que son los meses con pico de ocurrencias de esta especie en el Océano Atlántico Sudoccidental 
Tabla 1. Registros de las cuatro especies de focas citadas para Uruguay. CA: Canelones, CO: Colonia, MA: Maldonado, MO: Montevideo, RN: Río Negro, RO: Rocha, SA: Salto, SJ: San José, SO: Soriano.

\begin{tabular}{|c|c|c|c|c|}
\hline No. & Especie & Localidad & Fecha & Fuente \\
\hline 1 & H. leptonyx & Isla Verde, R0 & Enero 1970 & Ximénez \& Langguth, 2008 \\
\hline 2 & H. leptonyx & Punta Palmar, R0 & Agosto 1981 & Vaz Ferreira, 1984 \\
\hline 3 & H. leptonyx & Piriápolis, MA & 18 Setiembre 2000 & Naya \& Achaval, 2006 \\
\hline 4 & L. weddellii & Isla Rasa, R0 & Julio 1966 & Ximénez et al, 1972 (ZVCM 5732) \\
\hline 5 & L. carcinophaga & Puerto de Montevideo, MO & Previo a 1943 & $\begin{array}{l}\text { Pérez Fontana, 1943; Vaz Ferreira, } 1965 \\
\text { (MNHN 1302) }\end{array}$ \\
\hline 6 & L. carcinophaga & San Luis, CA & 27 Noviembre 2016 & Presente trabajo \\
\hline 7 & L. carcinophaga & Isla de Lobos, MA & Previo a 1943 & Pérez Fontana, 1943; Vaz Ferrerira, 1950 \\
\hline 8 & L. carcinophaga & Isla de Lobos, MA & Setiembre 1963 & Ximénez et al, 1972 \\
\hline 9 & L. carcinophaga & Punta del Este, MA & 13 Junio 2010 & Casas \& Arévalo, 2010 \\
\hline 10 & M. leonina & Salto Grande, SA & Década de 1980 & Olazarri, 1999 \\
\hline 11 & M. Ieonina & Nuevo Berlín, RN & Década de 1970 & Olazarri, 1999 \\
\hline 12 & M. Ieonina & Mercedes, RN & 19 Abril 2016 & Presente nota \\
\hline 13 & M. Ieonina & Colonia Concordia, SO & Agosto 1982 & Olazarri, 1999 \\
\hline 14 & M. Ieonina & Punta Riachuelo, CO & 29 Junio 1979 & Castello, 1984 \\
\hline 15 & M. Ieonina & Desconocida, CO & 1999 & Lewis et al., 2006 \\
\hline 16 & M. Ieonina & Arroyo Cufré, SJ & 2002 & Presente trabajo \\
\hline 17 & M. Ieonina & Arroyo Las Piedras, CA & Previo a 1970 & Vaz Ferreira, 1970 \\
\hline 18 & M. Ieonina & Santa Catalina, MO & 24 Abril 2015 & Presente trabajo \\
\hline 19 & M. Ieonina & Pocitos, MO & 1923 & MNHN 1277 \\
\hline 20 & M. Ieonina & Playa Trouville, MO & 8 Noviembre 1965 & Daciuk, 1973; Castello, 1984; González, 1986 \\
\hline 21 & M. Ieonina & Playa de los Ingleses, MO & 13 Junio 2006 & Presente trabajo \\
\hline 22 & M. Ieonina & Playa La Mulata, M0 & 2001 & Presente trabajo \\
\hline 23 & M. Ieonina & Isla de Flores, CA & 27 Junio 1973 & Presente trabajo \\
\hline 24 & M. Ieonina & El Pinar, CA & 8 Noviembre 2004 & Presente trabajo \\
\hline 25 & M. Ieonina & El Fortín, CA & 28 Abril 2004 & MNHN 5767 \\
\hline 26 & M. Ieonina & Punta del Este, MA & Diciembre 1999 & Presente trabajo \\
\hline 27 & M. Ieonina & Punta del Este, MA & Agosto 2002 & Presente trabajo \\
\hline 28 & M. Ieonina & Punta del Este, MA & 2004 & Presente trabajo \\
\hline 29 & M. Ieonina & Punta del Este, MA & 25 Junio 2010 & Presente trabajo \\
\hline 30 & M. Ieonina & Isla de Lobos, MA & 1921 & Smith, 1927 ( NMNH 239141) \\
\hline 31 & M. Ieonina & Isla de Lobos, MA & Previo a 1943 & Pérez Fontana, 1943 (2 individuos) \\
\hline 32 & M. Ieonina & Isla de Lobos, MA & Previo a 1950 & Vaz Ferreira, 1950 (3 individuos) \\
\hline 33 & M. Ieonina & Isla de Lobos, MA & Enero 1949 & Vaz Ferreira, 1950 \\
\hline 34 & M. leonina & Isla de Lobos, MA & 1954 a 1970 & $\begin{array}{l}\text { Ximénez et al, } 1972 \text { (16 individuos } \\
\text { registrados) }\end{array}$ \\
\hline 35 & M. Ieonina & Isla de Lobos, MA & 1961 & Vaz Ferreira, 1965 \\
\hline 36 & M. Ieonina & Isla de Lobos, MA & 1957 & Ximénez, 1964 \\
\hline 37 & M. Ieonina & Isla de Lobos, MA & 1959 & Ximénez, 1964 \\
\hline 38 & M. Ieonina & Isla de Lobos, MA & 2003 & $\begin{array}{l}\text { Lewis et al., } 2006 \text { (28 individuos } \\
\text { registrados) }\end{array}$ \\
\hline 39 & M. leonina & Isla de Lobos, MA & 2004 & $\begin{array}{l}\text { Lewis et al., } 2006 \text { (3 individuos } \\
\text { registrados) }\end{array}$ \\
\hline 40 & M. Ieonina & Isla de Lobos, MA & 2005 & Lewis et al., 2006 \\
\hline 41 & M. leonina & Isla de Lobos, MA & 2000 a 2016 & $\begin{array}{l}\text { Presente trabajo (varios individuos } \\
\text { registrados) }\end{array}$ \\
\hline
\end{tabular}


Tabla 1. (Continuación)

\begin{tabular}{|c|c|c|c|c|}
\hline No. & Especie & Localidad & Fecha & Fuente \\
\hline 42 & M. Ieonina & Manantiales, MA & Julio 2000 & MMPE 029 \\
\hline 43 & M. Ieonina & Barra de Maldonado, MA & Julio 1997 & Presente trabajo \\
\hline 44 & M. Ieonina & José Ignacio, MA & 3 Febrero 2003 & Daneri, 2009 \\
\hline 45 & M. leonina & La Paloma, RO & 2002 & Presente trabajo \\
\hline 46 & M. Ieonina & La Paloma, R0 & 1993 & Lewis et al., 2006 \\
\hline 47 & M. leonina & La Paloma , R0 & 2003 & Lewis et al., 2006 \\
\hline 48 & M. leonina & La Paloma, RO & 10 Enero 2005 & Daneri, 2009 \\
\hline 49 & M. Ieonina & Isla La Paloma, R0 & 2000 & Ximénez \& Langguth, 2008 \\
\hline 50 & M. Ieonina & Cabo Polonio, Ro & Previo a 1952 & Vaz Ferreira, 1952. \\
\hline 51 & M. leonina & Cabo Polonio, R0 & Febrero 2003 & Presente trabajo \\
\hline 52 & M. Ieonina & Cabo Polonio, RO & 5 Junio 2005 & Presente trabajo \\
\hline 53 & M. Ieonina & Cabo Polonio, R0 & 13 Noviembre 2007 & Presente trabajo \\
\hline 54 & M. leonina & Valizas, RO & 2004 & MNHN 5768 \\
\hline 55 & M. leonina & Islas de Torres y Castillos, RO & 1956 a 1970 & $\begin{array}{l}\text { Ximénez et al, } 1972 \text { (14 individuos } \\
\text { registrados) }\end{array}$ \\
\hline 56 & M. Ieonina & Isla Rasa, R0 & 1921 & Smith, 1927 \\
\hline 57 & M. Ieonina & Isla Rasa, R0 & 2007 & Presente trabajo \\
\hline 58 & M. leonina & Isla Rasa, R0 & 15 Agosto 2009 & Presente trabajo \\
\hline 59 & M. leonina & Isla del Marco, R0 & - & Ximénez \& Langguth, 2008 \\
\hline 60 & M. leonina & Isla Verde , RO & Enero 1969 & Pilleri, 1971; Pilleri \& Gihr, 1974 \\
\hline 61 & M. leonina & Isla Verde, RO & 14 Enero 2004 & Lewis et al., 2006 \\
\hline 62 & M. leonina & Isla Verde, RO & - & Ximénez \& Langguth, 2008 \\
\hline 63 & M. leonina & Isla Verde, RO & 9 Febrero 2010 & Presente trabajo \\
\hline 64 & M. Ieonina & Islote Coronilla, R0 & 1997 & Ximénez \& Langguth, 2008 \\
\hline
\end{tabular}

reportados por Rodríguez et al. (2003) y Moura et al. (2011).

La foca de Weddell presentó un único registro para Uruguay proveniente de la Isla Rasa (Rocha) en julio de 1966 (Ximénez et al., 1972). Este corresponde al mayor desplazamiento hacia el norte conocido para la especie en el Océano Atlántico (Rice, 1998; Bastida et al., 2007, Jefferson et al., 2015). El cráneo de este ejemplar permaneció perdido por varios años, hasta que fuera recientemente encontrado e ingresado a la colección de Zoología de Vertebrados de la Facultad de Ciencias como ZVCM 5732.

La primera referencia de foca de Weddell en Uruguay fue realizada por Smith (1927), registrando en el año 1921 algunos ejemplares en Isla de Lobos (Maldonado) y en Isla Rasa (Rocha). Smith (1927) asegura haber colectado una piel de $L$. weddellii en la Isla de Lobos (Maldonado) y llevada a Washington (Estados Unidos). El cráneo y la piel se conservan actualmente en la colección de mamíferos del National Museum of Natual History (Washington D.C) con el número NMNH 239141 y fue re-identificado como $M$. leonina (com. pers. Charles W. Potter) lo que deja sin efecto los registros de dicho autor.

La foca cangrejera presenta cinco registros, el primero proviene del Puerto de Montevideo (sin fecha, aunque anterior a 1943; Pérez Fontana, 1943) del cual se conserva el cráneo en la colección del Museo Nacional de Historia Natural con número MNHN 1302. Otros dos provienen de la Isla de Lobos (Maldonado), uno cuya fecha se desconoce (Pérez Fontana, 1943; Vaz Ferreira, 1950), mientras que el otro fue registrado en setiembre de 1963 (Ximénez et al., 1972). Un registro proviene de Punta del Este (Maldonado) el 13 de junio de 2010, de este ejemplar no se conserva material en colecciones ya que fue rehabilitado y liberado días después (Casas \& Arévalo, 2010). Otro registro proviene de San Luis (Canelones) el 27 de setiembre de 2016. Los tres registros con fecha conocida (o aproximada) de foca cangrejera en Uruguay concuerdan con lo reportado por Olivera et al. (2006) para Brasil, quienes encuentran que los registros de esta especie ocurren mayormente durante el verano y otoño, así como en los meses de invierno.

El elefante marino del Sur es el fócido más frecuente en Uruguay, registrándose casi todos los años la 
llegada de algún individuo (González, 2001) con apariciones de ejemplares durante las cuatro estaciones del año (Ponce de León, 2000). Esta especie presenta registros en todos los departamentos costeros (desde Rocha hasta Colonia), existiendo registros en sistemas de agua dulce sobre el Río Uruguay en los departamentos de Soriano, Rio Negro y Salto (Vaz Ferreira, 1970; Castello, 1984; Olazarri, 1999, presente trabajo). La mayor cantidad de registros para esta especie se da en Isla de Lobos, lo que es esperable, ya que Lewis et al. (2006) reconocen a esa isla como una de las dos áreas tradicionales de descanso para esta especie en el Océano Atlántico.

Aunque en Uruguay no existen colonias reproductivas se han registrado partos de elefante marino (Vaz Ferreira, 1950, 1952; Ximénez, 1964; Ximénez \& Langguth, 2002; 2008) de los cuales en un caso se trató de mellizos (Ximénez \& Langguth, 2008). Sin embargo estos serían fenómenos aislados y no representarían una expansión de la colonia (Lewis et al., 2006). A su vez, las aguas territoriales uruguayas pertenecen al área potencial de distribución estimada por Lewis et al. (2006) para los individuos de la colonia reproductiva de Península Valdéz (Argentina).

Las variables ambientales como vientos y corrientes marinas, los desplazamientos en busca de alimento y el crecimiento poblacional de algunas de las especies podrían influir en la dispersión de algunos especímenes a regiones lejanas de sus colonias reproductivas como lo señalan Magalhães et al. (2003), Silva (2004) y Moura et al. (2010). Asimismo, el registro sistemático de la ocurrencia de especímenes de fócidos contribuye a un mejor entendimiento de los patrones de dispersión y desplazamientos de estas especies.

Se agradece a Pablo Etchegaray (Museo del Mar, Punta del Este), Melitta Meneghel y Daniel Hernández (Zoología de Vertebrados, Facultad de Ciencias) quienes amablemente permitieron el acceso a las colecciones, Giancarlo Liparulo (Muséum d'histoire naturelle, Geneva) por el apoyo bibliográfico, a Charles W. Potter (National Museum of Natural History, Washington D.C) por facilitar la información de ejemplares en el National Museum of Natural History. A los compañeros del Laboratorio de Mamíferos Marinos del Museo Nacional de Historia Natural: Meica Valdivia, Sabrina Riverón, y Alfredo Le Bas por su permanente apoyo.

\section{REFERENCIAS}

Achaval F., Clara M. \& Olmos A. 2008. Mamíferos de la República Oriental del Uruguay, $2^{\mathrm{a}}$ edición. Zonalibro Industria Gráfica, Montevideo. 216 pp.

Bastida R. \& Rodríguez D. 2006. Otariidae, Phocidae. En R.M. Barquez, M.M. Díaz \& R.A. Ojeda.
(Eds.). Mamíferos de Argentina. Sistemática y Distribución, pp. 107-113. Sociedad Argentina para el Estudio de los Mamíferos, Tucumán.

Bastida R., Rodríguez D., Secchi E. \& da Silva V. 2007. Mamíferos Acuáticos de Sudamérica y Antártida, 1 a edición. Vázquez Mazzini, Buenos Aires. 368 pp.

Bastos B.L., Norberto G.O., Maia-Nogueira R. \& Guimarães J.E. 2006. Avaliação hematológica e dosagem bioquímica de ALT, AST e creatinina em elefante-marinho-do-sul, Mirounga leonina (Linnaeus, 1758), encontrado no litoral de Salvador, Bahia. Brazilian Journal of Veterinary Research and Animal Science, 43: 18-23.

Berg C. 1898. Lobodon carcinophagus (H. J.) Gr. en el Río de la Plata. Comunicaciones del Museo Nacional de Buenos Aires, 1(15): 93.

Berta A., Sumich J.L. \& Kovacs K.M. 2006. Marine Mammals: Evolutionary Biology, 2nd Edition. Elsevier, London. 547 pp.

Carvalho C.T. 1975. Ocorrência de mamíferos marinhos no Brasil. Boletim Técnico do Instituto Florestal, 16:13-32.

Casas L. \& Arévalo A.P. 2010. Registro y rehabilitación de Lobodon carcinophaga (Mammalia, Pinnipedia, Focidae) en la costa de Uruguay. Resúmenes del Primer Congreso Uruguayo de Zoología. Sociedad Zoológica del Uruguay, Montevideo, pp.163.

Castello H. P. 1984. Registros de elefante marino, Mirounga leonina (Carnivora, Phocidae) en las costas del Atlántico S.O., fuera del área de cría. Revista del Museo Argentino de Ciencias Naturales "Bernardino Rivadavia" (Zoología), 13: $235-243$.

Castello H.P. \& Rumboll M. 1978. Extension of range of the leopard seal, Hydrurga leptonyx, for the Argentine coast. Mammalia, 42: 135-137.

Cherem J.J., Simões-Lopes P.C., Althoff S. \& Graipel M.E. 2004. Lista dos mamíferos do Estado de Santa Catarina, sul do Brasil. Mastozoología Neotropical, 11: 151-185.

Daciuk C. 1973. Notas faunísticas y bioecológicas de la Península Valdez y Patagonia. X. Estudio cuantitativo y observaciones del comportamiento de la población del elefante marino del sur Mirounga leonina (Linne) en sus apostaderos de la provincia de Chubut (República Argentina). Physis (Sección C), 32: 403-422.

Daneri G.A. 2009. Two records of male southern elephant seals (Mirounga leonina) on the Atlantic coast of Uruguay. Latin American Journal of Acuatic Mammals 7(1-2): 101-102.

Daneri G.A., Varela E.A., Di Martino C.C., Harrington A. \& Montiel R.A. 2011. Atypical records of pinniped specimens housed in the Museo Argentino de Ciencias Naturales. Latin American 
Journal of Aquatic Mammals, 9: 150-153.

Drehmer C.J., Ferigolo J. \& Borsato E.S. 1998. Ocorrência de Mirounga leonina Linnaeus (Pinnipedia, Phocidae) no extremo-sul do Brasil: agressão e patologias. Revista Brasileira de Zoologia, 15: 1061-1068.

Ferreria H.D.O., Zerbini A.N. \& Siciliano, S. 1995. Occurrence of crabeater and leopard seals in Southern Brazil. En: Abstracts XI Biennial Conference on the Biology of Marine Mammals, 14-18 December 1995, Orlando, USA, pp 36.

González E.M. 2001. Guía de campo de los mamíferos de Uruguay. Introducción al estudio de los mamíferos. Vida Silvestre, Montevideo. 339 pp.

González E.M. \& Martínez J.A. 2010. Mamíferos de Uruguay. Guía de Campo e introducción a su estudio y conservación. Banda Oriental, Montevideo. 464 pp.

González J.C. 1986. Los pinnípedos del Uruguay. Almanaque del Banco de Seguros del Estado. Banco de Seguros del Estado, 69: 36-41.

Jefferson T.A., Webber M.A. \& Pitman R.L. 2015. Marine Mammals of the World: a comprehensive guide to their identification. 2nd edition. Academic Press, San Diego. 616 pp.

Lewis M., Campagna C., Martin M.R. \& Fernández T. 2006. Southern elephant seals north of the Antarctic Polar Front. Antarctic Science, 18: 213-221.

Lewis M. Campagna C., Quintana F. \& Falabella V. 1998. Estado actual y distribución de la población del elefante marino del sur en la Península Valdés, Argentina. Mastozoología Neotropical, 5: 29-40.

Lodi L., Mayerhofer L.C., Farias Júnior S.G. \& Cruz F.S. 2005. Nota sobre la ocurrência de foca caranguejeira Lobodon carcinophagus (Hombron \& Jacquinot, 1842) (Mammalia: Pinnipedia), no estado do Río de Janeiro, Brasil. Biotemas, 18: 151-161.

Lodi L. \& Siciliano S. 1989. A southern elephant seal in Brazil. Marine Mammal Science, 5: 313.

Magalhães F.A., Hassel, L.B., Venturotti, C. \& Sicialiano, S. 2003. Southern elephant seals (Mirounga leonina) on the coast of Rio de Janeiro State. Latin American Journal of Aquatic Mammals, 2: 55-56.

McMahon C.R., Bester M.N., Burton H.R., Hindell M.A. \& Bradshaw C.J.A. 2005. Population status, trends and a re-examination of the hypotheses explaining the recent declines of the southern elephant seal Mirounga leonina. Mammal Review, 35: 82-100.

Moura J.F., Di Dario B.P.S., Lima L.M. \& Siciliano S. 2010. Southern elephant seals (Mirounga leonina) along the Brazilian coast: review and additional records. Marine Biodiversity Records, 3(e18): 1-5.
Moura J.F., Di Dario B.P.S. \& Siciliano S. 2011. Occurrence of pinnipeds on the coast of Rio de Janeiro State, Brazil. Marine Biodiversity Records, 4(e27): 1-10.

Naya D.E. \& Achaval F. 2006. Nuevos registros de especies poco comunes de Pinnipedia y primer registro de Arctocephalus gazella (Peters, 1875) (Pinnipedia; Otaridae) para el Uruguay. Boletín de la Sociedad Zoológica del Uruguay, 15: 2327.

Olazarri J. 1999. Ingreso de Pinnipedia (Mammalia) en aguas dulces del bajo Río Uruguay y sus afluentes. Boletín de la Sociedad Zoológica del Uruguay, 10: 106-108.

Oliveira A., Kolesnikovas C.K.M., Serafini P.P., Moreira L.M.P., Pontalti M., Simões-Lopes P.C., \& Barreto A.S. 2011. Occurrence of pinnipeds in Santa Catarina between 2000 and 2010. Latin American Journal of Aquatic Mammals, 9: 145149.

Oliveira L.R., Machado R., Meller Alievi M. \& Würdig N.L. 2006. Crabeater seal (Lobodon carcinophaga) on the coast of Rio Grande do Sul State, Brazil. Latin American Journal of Acuatic Mammals, 5: 145-148.

Pérez Fontana H. 1943. Informe sobre la industria lobera (ciento diez años de explotación de la industria lobera en nuestro país). Servicio Oceanográfico y de Pesca, Montevideo. 69 pp.

Pilleri G. 1971. Biologisch-anatomische Forschungsreise nach Uruguay zum Studium des La-Plata-Delphins Pontoporia blainvillei. Verlag Hirnanatomisches Institut, Ostermundigen, Bern. 28pp.

Pilleri G. \& Gihr M. 1974. Record of a Southern Elephant Seal (Mirounga leonina Linnaeus) off the Isla Verde, Uruguay. Revue Suisse de Zoologie, 81: 95-100.

Pinedo M.C. 1990. Ocorrência de Pinípedes na costa brasileira. Garcia de Orta (Série Zoológica), 15: 37-48.

Ponce de León A. 2000. Taxonomía, sistemática y sinopsis de la biología y ecología de los pinipedios del Uruguay. En M. Rey \& F. Amestoy (Eds.) Sinopsis de la biología y ecología de las poblaciones de lobos finos y leones marinos de Uruguay. Pautas para su manejo y administración. Parte I. Biología de las especies, pp. 9-36. Proyecto URU/92/003. Instituto Nacional de Pesca - Programa de las Naciones Unidas para el Desarrollo, Montevideo.

Rice D.W. 1998. Marine Mammals of the World. Systematics and Distribution. Special Publication № 4. The Society for Marine Mammalogy, Lawrence, Kansas, United States of America. 231 pp.

Rodríguez D., Bastida R., Morón S., Rodríguez S. \& Loureiro J. 2003. Occurrence of leopard seal in 
Northern Argentina. Latin American Journal of Aquatic Mammals, 2: 51-54.

Rosas F.C.W., Capistrano L.C., Di Beneditto A.P. \& Ramos, R. 1992. Hydrurga leptonyx recovered from the stomach of a tiger shark captured off Rio de Janeiro coast, Brazil, Mammalia, 56: 153-155.

Scolaro J.A. 1976. Nota sobre una foca cangrejera (Lobodon carcinophaga) capturada en Puerto Madryn, Chubut. Neotropica, 22: 117-119.

Siciliano S. \& Lodi L. 1989. Pinnipeds observed along the coast of Rio de Janeiro State, Brasil 19871989. En Abstracts VIII Biennial Conference on the Biology of Marine Mammals, 7-11 December 1989, California, USA, pp 62.

Silva K.G. 2004. Os pinípedes no Brasil. Ocorrências, estimativas populacionais e conservação. PhD Thesis, Fundação Universidade Federal do Rio Grande, Rio Grande. 242 pp.

Simões-Lopes P.C., Drehmer C.J. \& Ott P.H. 1995. Nota sobre os Otariidae e Phocidae (Mammalia: Carnivora) da costa norte do Rio Grande do Sul e Santa Catarina, Brasil. Biociências, 3: 173-181.

Smith H.M. 1927. The Uruguayan fur-seal islands. Zoologica, 9: 271-294.

Stirling I. 1971. Leptonychotes weddellii. Mammalian Species. 6: 1-5.

Vaz Ferreira R. 1950. Observaciones sobre la Isla de Lobos. Revista de la Facultad de Humanidades y Ciencias, 5: 145-176.
Vaz Ferreira R. 1952. Observaciones sobre las Islas de Torres y de Castillo Grande. Revista de la Facultad de Humanidades y Ciencias, 9: 237258.

Vaz Ferreira R. 1965. Ecología terrestre y marina de los pinnípedos del Atlántico sudoccidental. Anais da Academia Brasileira de Ciencias, 37: 179191.

Vaz Ferreira R. 1970. Fauna: conservación y recursos. Nuestra Tierra, 45: 1-60.

Vaz Ferreira R. 1984. La foca leopardo Hydrurga leptonyx (de Blainville, 1820) (Pinnipedia, Phocidae) en el Uruguay. Boletín de la Sociedad Zoológica del Uruguay, 2: 18-21.

Ximénez A., Langguth A. \& Praderi R. 1972. Lista sistemática de los mamíferos del Uruguay. Anales del Museo Nacional de Historia Natural de Montevideo, 7: 1-49.

Ximénez I. 1964. Estudio preliminar de la distribución geográfica actual de los pinnípedos en América Latina. Boletín del Instituto de Biología Marina, 7: $65-72$.

Ximénez I. \& Langguth E. 2002. Isla de Lobos. Graphis, Montevideo. $231 \mathrm{pp}$.

Ximénez I. \& Langguth E. 2008. Islas y cabos atlánticos del Uruguay, Paideia Ediciones, Montevideo. $292 \mathrm{pp}$. 